\title{
A Differentially Coherent PN Code Acquisition Receiver for CDMA Systems
}

\author{
Mohammad H. Zarrabizadeh and Elvino S. Sousa, Senior Member, IEEE
}

\begin{abstract}
New differentially coherent detectors for acquisition of direct sequence spread-spectrum signals are introduced. These detectors are alternatives to the noncoherent detectors that have been considered almost exclusively in the past. The proposed detectors are suitable for commercial code-division multiple-access (CDMA) systems which operate with a relatively large noise floor and provide a surprisingly large signal-to-noise ratio (SNR) improvement over the noncoherent detectors of approximately $5 \mathrm{~dB}$. Under the random code sequence assumption, an exact analysis of the differentially coherent detection performance for both full period correlation (FPC) and partial period correlation (PPC) is carried out. The detector performance in terms of detection and false alarm probabilities for both partial and full period correlations is investigated, and the results are compared with those of classical noncoherent detection. The mean acquisition time for both single-dwell and multiple-dwell acquisition schemes are compared with their noncoherent counterparts.
\end{abstract}

Index Terms - CDMA, differential coherent, PN code acquisition, spread spectrum, spreading code acquisition, synchronization.

\section{INTRODUCTION}

A CQUISITION of direct-sequence (DS) binary phase-shift keying (BPSK) spread-spectrum waveforms is achieved through coherent or noncoherent correlation. Due to low predespreading signal-to-noise ratio (SNR), the receiver must acquire the pseudorandom (PN) code before carrier synchronization. Thus far, the most common detector found in acquisition systems for DS receivers is the noncoherent detector, which may be comprised of a band-pass filter followed by a matched filter and a square-law envelope detector [1]. In this paper, we propose a differentially coherent acquisition receiver. This receiver greatly improves the detection and false alarm probabilities which makes it suitable for code-division multiple-access (CDMA) systems with large noise floors.

The acquisition receiver can be implemented using either an "active" correlator, where the correlation is performed sequentially on a "chip-by-chip" basis, or a "passive" correlator

Paper approved by G. L. Stuber, the Editor for Spread Spectrum of the IEEE Communications Society. Manuscript received March 1, 1996; revised February 23, 1997. This work was supported by a grant from the Canadian Institute for Telecommunications Research under the NCE Program of the Government of Canada. This paper was presented in part at the 1995 International Conference on Communications (ICC'95), Seattle, WA, June 1995 and at the Vehicular Technology Conference (VTC'95), Chicago, IL, July 1995.

M. H. Zarrabizadeh is with Lucent Technologies, Holmdel, NJ 07733-3030 USA.

E. S. Sousa is with the Department of Electrical and Computer Engineering, University of Toronto, Toronto, Ont., M5S 3G4 Canada.

Publisher Item Identifier S 0090-6778(97)08208-1. (or matched filter), where the correlation is performed on $M$ chips in parallel [4]. We consider the latter scheme throughout this paper, due to its faster acquisition process.

The matched filter acquisition scheme utilizing noncoherent detection has been investigated in [2], [3], and [5]. Basic acquisition parameters such as detection and false alarm probabilities, as well as the mean acquisition time have been calculated. As in noncoherent detection, differentially coherent detection does not need prior knowledge of the carrier phase and, therefore, it can be regarded as a potential substitute for the noncoherent technique which yields poor performance in the presence of heavy background noise or various sources of interference. A differentially coherent detection scheme has been considered in [10], and its performance in the presence of white Gaussian noise was investigated. It was also shown that, for data detection, a generalized differentially coherent detector outperforms noncoherent schemes in the presence of self noise and interpath interference [7]. We assume $H_{1}$ as the hypothesis that the codes are synchronized and $H_{0}$ as the alternative hypothesis which corresponds to code misalignment. Under hypothesis $H_{0}$, in a differentially coherent detector, the decision variable is the product of consecutive uncorrelated samples, whereas, in a noncoherent detector, the decision variable is the square of a single sample. This characteristic of the differentially coherent detection scheme suggests a significant improvement in the performance of the differential detection scheme over the noncoherent scheme [8], [9].

In addition to the additive white Gaussian noise (AWGN), the matched filter output also contains a component due to the autocorrelation of the PN code which we refer to as the code self noise. The autocorrelation self noise is usually modeled as a Gaussian random variable [3]. We also adopt this assumption in this paper. It is well known that the multiuser interference in CDMA systems can be modeled as a Gaussian random process for a large number of users [11]. Thus, all the results in this paper can be directly applied to CDMA systems.

Depending upon the duration of observation, the acquisition detectors can be differentiated according to whether they utilize partial period correlation (PPC) or full period correlation (FPC). With FPC, the correlation is performed over a full code period, whereas the correlation is carried out over a segment of the long PN sequence using PPC [1]. While the detection performances of both PPC and FPC are similar in noncoherent detection, they differ in the differential case. With noncoherent detection, the decision variable is the envelope of the noncoherent correlator output. Considering 


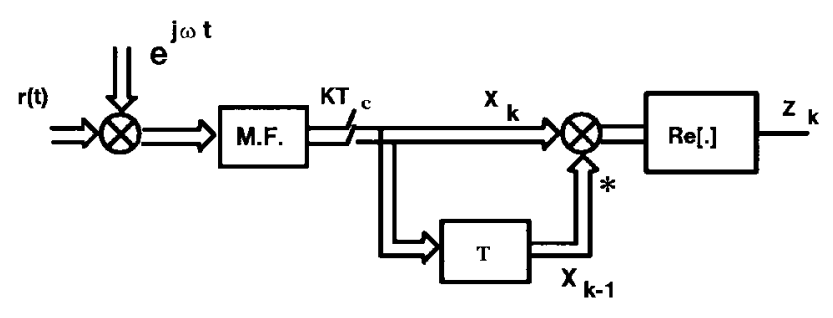

Differential FPC detector

(a)

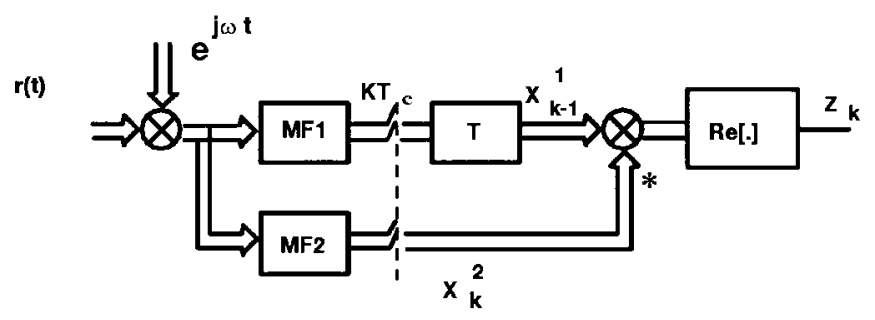

Differential PPC detector

(b)

Fig. 1. Receiver block diagrams.

long random codes for both PPC and FPC, the statistics of the decision variables are the same for the two detection techniques. With the differential detection technique, however, the self-noise component of the consecutive samples, the product of which forms the decision variable, are the same in FPC, whereas they are independent random variables in PPC. Furthermore, unlike with noncoherent detection, the thermal noise components of the multiplicative samples are statistically independent in both differential cases. Thus, we expect that the differential technique outperforms the noncoherent one in terms of detection, false alarm probability, and mean acquisition time.

The paper is organized as follows. Section II introduces the system model. In Section III, a DS acquisition receiver utilizing differentially coherent FPC is analyzed. The acquisition performance, in terms of detection and false alarm probabilities, is derived. In Section IV, the same analysis for differentially coherent PPC detection is carried out, and the performance is compared with the FPC detection scheme. Based upon the results in Sections III and IV, in Section V, the mean acquisition times in single- and multiple-dwell detection algorithms are evaluated for both PPC and FPC schemes, and the results are compared with the classical noncoherent scheme. In Section VI, the overall acquisition performances for all different detection and decision schemes are illustrated, and the results are discussed. Section VII concludes the paper.

\section{SySTEM MODEL}

The receiver structures for the proposed differentially coherent PPC and FPC acquisition schemes are shown in Fig. 1(a) and (b), respectively. The receivers can be realized utilizing typical in-phase and quadrature (I-Q) matched filters followed by two multipliers and two delay lines, as shown in Fig. 2. Without loss of generality, we can assume that the two codes are synchronized at the chip level, and the detector output is sampled every chip period. Similar comparison between the two detection schemes can be obtained for higher sampling rates. Each sample is compared with a given threshold, which is set by a conventional threshold setting algorithm [6]. If the sample exceeds the threshold, then the receiver either accepts the corresponding phase of the PN code and goes into tracking mode, in the case of a single-dwell detector, or goes into the verification mode of the acquisition scheme in the case of a multiple-dwell detector. In the verification mode, only the specific phase position which was previously decided upon in the search mode will be examined further and $A$ independent threshold comparisons are performed. If at least $B$ out of $A$ of these exceed the threshold, then acquisition is declared, and the receiver moves to the tracking mode. Otherwise, it rejects the code phase, and a new search is resumed.

In the verification mode of the multiple-dwell scheme with FPC detection, the receiver advances the local code at the same rate as the incoming code. In the PPC case, however, the reference code for the second matched filter is delayed by $M T_{c}$ seconds with respect to the first one during the search mode. In verification mode, the first matched filter local code is run in parallel with the incoming code, while the reference code of the second matched filter is delayed by $M T_{c}$ with respect to the input signal.

In this paper, both single- and multiple-dwell schemes are examined, and their performances for differential and noncoherent detectors are investigated. We assume timing uncertainty of one full code period and the simple straight serial search strategy [3] in our acquisition method. In the analysis, we consider a decision time of one chip period.

\section{DifFEREnTIALly COHERENT FPC}

The receiver structure for a differentially coherent DS/BPSK utilizing FPC is shown in Fig. 1(a). The complex representation of the signal at the front end of the acquisition receiver is

$$
r(t)=\sqrt{\frac{E_{c}}{T_{c}}} c\left(t+\beta T_{c}\right) \mathrm{e}^{j(\omega t+\theta)}+N(t)
$$

where $\beta$ is the received code phase offset which is assumed to be an integer, $c(t)$ is the PN waveform, $\theta$ is the carrier phase, $T_{c}$ is the chip period, $E_{c}$ is the received chip energy, and $N(t)$ is a complex white Gaussian noise process with twosided power spectral density $N_{0} / 2$. The output samples of the matched filter are given by

$$
X_{k}=Y_{k} \mathrm{e}^{j \theta}+N_{k}^{\prime}
$$

where

$$
Y_{k}=\sqrt{\frac{E_{c}}{T_{c}}} \int_{0}^{M T_{c}} c(t) c\left(t+\beta T_{c}+k T_{c}\right) d t
$$

and

$$
N_{k}^{\prime}=\int_{0}^{M T_{c}} c(t) N\left(t+k T_{c}\right) \mathrm{e}^{-j \omega t} d t .
$$

$N_{k}$ is a complex Gaussian noise sample with variance $\sigma_{n}^{2}=$ $\frac{N_{0} M T_{c}}{2}$. Under the hypothesis $H_{0}$ and assuming $c(t)$ as a 


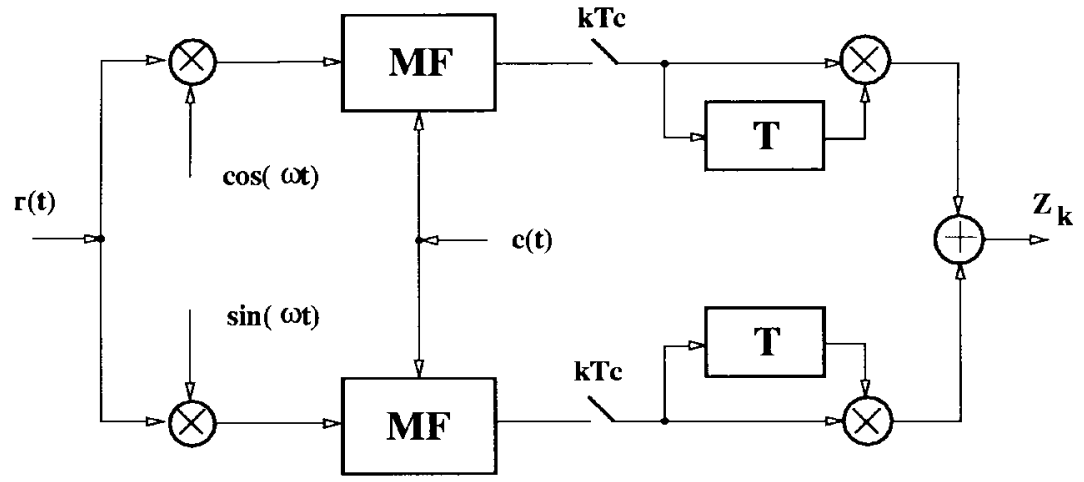

Fig. 2. I-Q receiver block diagram for FPC.

random sequence, $Y_{k}$ is a binomially distributed random variable with the probability density function given by

$$
P_{Y}(y)=2^{-M} \sum_{n=0}^{M}\left(\begin{array}{c}
M \\
n
\end{array}\right) \delta\left[y-\sqrt{E_{c} T_{c}}(2 n-M)\right]
$$

where $\delta(\cdot)$ is the Dirac delta function. According to the central-limit theorem, if the integrations are performed over many chips $(M \gg 1)$, which is usually the case in practice, the distribution of $Y_{k}$ can be approximated by a zero-mean Gaussian distribution with variance $\sigma_{Y}^{2}=E_{c} M T_{c}$. The decision variable $Z_{k}$ is given by

$$
Z_{k}=\operatorname{Re}\left[\left(Y_{k} \mathrm{e}^{j \theta}+N_{k}^{\prime}\right)\left(Y_{k-M} \mathrm{e}^{j \theta}+N_{k-M}^{\prime}\right)^{*}\right] .
$$

Clearly, for FPC, $Y_{k}=Y_{k-M}$, and $N_{k}^{\prime}$ and $N_{k-M}^{\prime}$ are two independent complex Gaussian random variables. By absorbing $\theta$ in the phases of the complex noise components, the decision variable is simplified to

$$
Z_{k}=\left(Y_{k}+N_{k}^{R}\right)\left(Y_{k}+N_{k-M}^{R}\right)+N_{k}^{I} N_{k-M}^{I} .
$$

We now derive the statistics of the two multiplicative terms in the right-hand side of (7). The first term is the product of two dependent Gaussian random variables

$$
x=Y_{k}+N_{k}^{R} \quad y=Y_{k}+N_{k-M}^{R} .
$$

It is seen that $x-y$ and $x+y$ are two independent Gaussian r.v. with variances $\sigma_{1}^{2}=2 \sigma_{n}^{2}$ and $\sigma_{2}^{2}=2 \sigma_{n}^{2}+4 \sigma_{Y}^{2}$, respectively. Therefore, the joint probability density function (pdf) for $x$ and $y$ is given as

$$
\begin{aligned}
f_{x, y}(x, y)= & \frac{1}{2 \pi \sigma_{n} \sqrt{2 \sigma_{Y}^{2}+\sigma_{n}^{2}}} \\
& \cdot \exp \left(-\frac{(x-y)^{2}}{4 \sigma_{n}^{2}}-\frac{(x+y)^{2}}{8 \sigma_{Y}^{2}+4 \sigma_{n}^{2}}\right) .
\end{aligned}
$$

The density of the r.v. $z=x y$ is given by [13]

$$
f_{1}(z)=\int_{-\infty}^{\infty} \frac{1}{|w|} f_{x, y}\left(w, \frac{z}{w}\right) d w .
$$

It is shown in the Appendix that $f_{1}(z)$ can be expressed in the following closed form:

$$
\begin{aligned}
f_{1}(z)= & \frac{1}{\pi \sigma_{n}^{2} \sqrt{1+2 \rho^{2}}} \\
& \cdot \exp \left(\frac{\rho^{2} z}{\sigma_{n}^{2}\left(1+2 \rho^{2}\right)}\right) K_{0}\left(\frac{\left(1+\rho^{2}\right)|z|}{\sigma_{n}^{2}\left(1+2 \rho^{2}\right)}\right)
\end{aligned}
$$

where $\rho=\frac{\sigma_{Y}}{\sigma_{\eta}}=\sqrt{\frac{2 E_{c}}{N_{0}}}$ and $K_{0}(\cdot)$ is the modified Hankel function of order zero. The pdf for the second term in (7) is a special case of (11) with $\rho=0$, i.e., when the self-noise term vanishes; thus, its density function is given by

$$
f_{2}(z)=\frac{1}{\pi \sigma_{n}^{2}} K_{0}\left(\frac{|z|}{\sigma_{n}^{2}}\right) \text {. }
$$

The pdf for the decision variable $Z_{k}$ is given by

$$
f_{H_{0}}(z)=f_{1}(z) * f_{2}(z)
$$

where " *" denotes convolution. After some manipulation, the probability of false alarm is given by

$$
\begin{aligned}
P_{\mathrm{FA}}= & \int_{V_{T}}^{\infty} f_{H_{0}}(z) d z \\
= & \int_{-\infty}^{\infty} f_{1}(z) \int_{V_{T}-z}^{\infty} f_{2}(\beta) d \beta d z \\
= & \frac{1}{\pi \sqrt{1+2 \rho^{2}}} \int_{-\infty}^{\infty} \exp \left(\frac{\rho^{2} z}{1+2 \rho^{2}}\right) \\
& \cdot K_{0}\left(\frac{\left(1+\rho^{2}\right)|z|}{\left(1+2 \rho^{2}\right)}\right) \Phi\left(V_{T_{n}}-z\right) d z
\end{aligned}
$$

where

$$
\Phi(\alpha)=\frac{1}{\pi} \int_{\alpha}^{\infty} K_{0}(|t|) d t
$$

and $V_{T_{n}}=V_{T} / \sigma_{n}^{2}$ is the normalized threshold level. The integral in (16) can be obtained in closed form [15]

$$
\begin{aligned}
\Phi(\alpha)= & \frac{1}{2}-\left[\frac{1}{\pi} \alpha K_{0}(\alpha)\right. \\
& \left.+\frac{1}{2} \alpha\left\{L_{0}(\alpha) K_{1}(\alpha)+L_{1}(\alpha) K_{o}(\alpha)\right\}\right] .
\end{aligned}
$$

$K_{1}(\alpha)$ is the modified Hankel function of first order and $L_{0}(\alpha)$ and $L_{1}(\alpha)$ are Struve functions [15].

For large $E_{c} / N_{0}$, i.e., for $\rho$ comparable or greater than one, we can ignore the second multiplicative term in the decision variable. From (11), the asymptotic false alarm probability for high SNR values simplifies to

$$
P_{\mathrm{FA}_{\mathrm{Asymp}}}^{H} \approx \frac{\sqrt{1+2 \rho^{2}}}{\pi\left(1+\rho^{2}\right)} \cdot \int_{v^{*}}^{\infty} \exp \left(\frac{\rho^{2} z}{\left(1+\rho^{2}\right)}\right) K_{0}(z) d z
$$




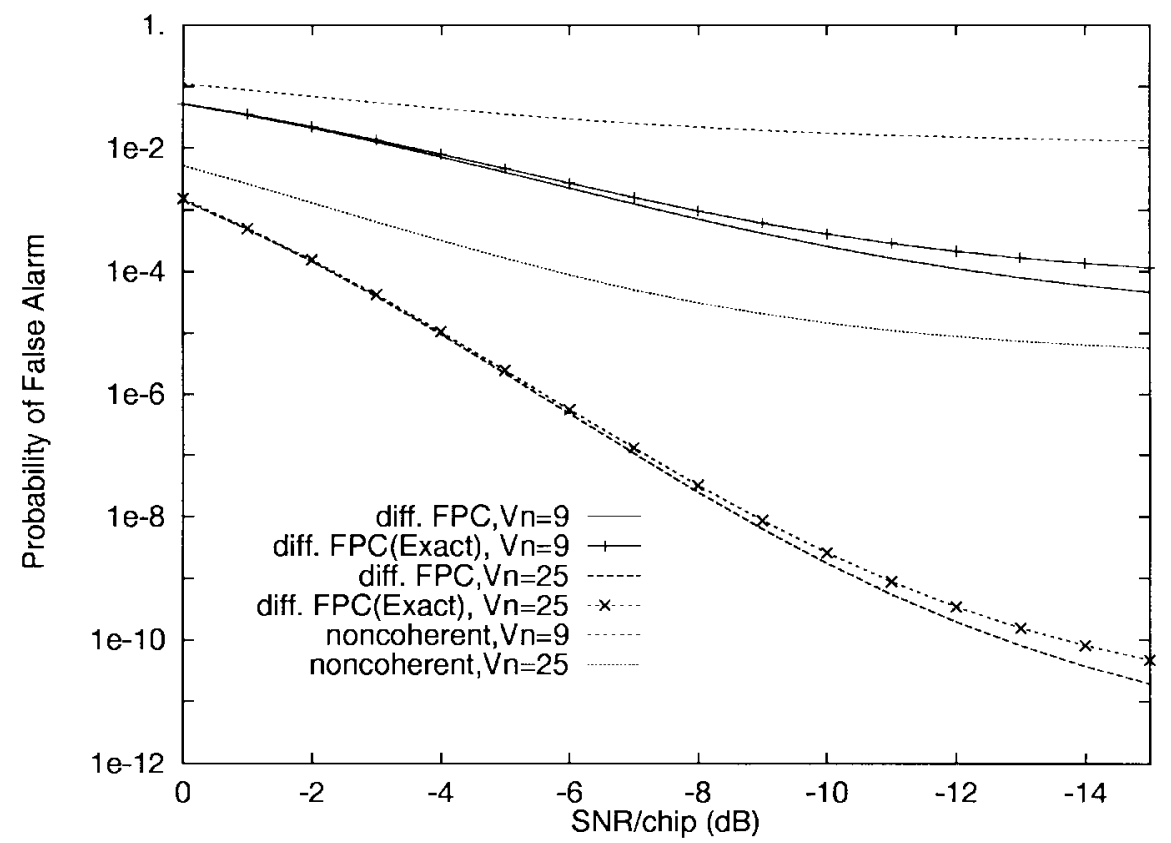

Fig. 3. False alarm probability versus chip SNR for FPC.

where

$$
v^{*}=\frac{\left(1+\rho^{2}\right)}{\left(1+2 \rho^{2}\right)} V_{T_{n}} .
$$

For low $E_{c} / N_{0}$, i.e., when $\rho \ll 1$, the self-noise component of the first term can be ignored. As shown in the Appendix, the density of the decision variable is given by

$$
\begin{aligned}
f_{H_{0}}(z) & \approx \frac{1}{\pi \sigma_{n}^{2}} K_{0}\left(\frac{|z|}{\sigma_{n}^{2}}\right) * \frac{1}{\pi \sigma_{n}^{2}} K_{0}\left(\frac{|z|}{\sigma_{n}^{2}}\right) \\
& =\frac{1}{2 \sigma_{n}^{2}} \exp \left(-\frac{|z|}{\sigma_{n}^{2}}\right) .
\end{aligned}
$$

From (20), the false alarm probability for very low SNR values can be written as

$$
P_{\mathrm{FA}_{\mathrm{Asymp}}}^{L} \approx \frac{1}{2} \exp \left(-\left|V_{T_{n}}\right|\right)
$$

With noncoherent detection, the density function of the decision variable under hypothesis $H_{0}$ is given by [3]

$$
\begin{aligned}
f_{H_{0}}(z)= & \frac{1}{2 \sigma_{n}^{2} \sqrt{1+\rho^{2}}} \\
& \cdot \exp \left(-\frac{z}{4 \sigma_{n}^{2}} \frac{2+\rho^{2}}{1+\rho^{2}}\right) I_{0}\left(\frac{z}{4 \sigma_{n}^{2}} \frac{\rho^{2}}{1+\rho^{2}}\right)
\end{aligned}
$$

and the corresponding false alarm probability becomes

$$
P_{\mathrm{FA}}=\frac{2 \sqrt{1+\rho^{2}}}{2+\rho^{2}} \int_{C^{*}}^{\infty} \exp (-z) I_{0}\left(\frac{\rho^{2}}{2+\rho^{2}} z\right) d z
$$

where

$$
c^{*}=\frac{V_{T_{n}}}{4} \frac{2+\rho^{2}}{1+\rho^{2}} .
$$

and $V_{T_{n}}$ has the previous definition. For lower values of SNR, $\rho$ tends to zero, and the false alarm probability is approximated by

$$
P_{\mathrm{FA} \text { Asymp. }}^{L} \approx \int_{\frac{V_{T_{n 2}}}{2}}^{\infty} \exp (-z) d z=\exp \left(-\frac{V_{T_{n}}}{2}\right) .
$$

It is seen that the arguments in the exponentials in (25) and (21) differ by a factor of two. This translates into a major difference in false alarm probability between the noncoherent and differential techniques.

As the asymptotic detection probabilities associated with the two detection schemes converge in a higher SNR range for the same threshold levels, a comparison between the two probabilities with the same detection thresholds is justified.

In Fig. 3, $P_{\mathrm{FA}}$ is plotted for both noncoherent and differentially coherent detection and for two normalized thresholds, as a function of the chip energy to noise power spectral density. From Fig. 3, we observe that there is a very small difference between the exact and approximated results in the differential case, especially in higher SNR values. The differential and noncoherent false alarm probability curves converge for large $E_{c} / N_{0}$. This is expected, since, in that region, the independent thermal noise components are negligible compared to the self noise. With FPC, the self-noise components of the consecutive samples are fully correlated, and the performance is determined by these dominant components. Hence, the false alarm probability curves converge for large SNR values.

In the more interesting range of SNR and false alarm probability, however, there is a significant difference between the two curves. In this area, statistically independent thermal noise components determine the false alarm probability and, therefore, the differential detection outperforms the classical noncoherent detection. It is seen from Fig. 3 that, for certain threshold values, the false alarm probability is four to five orders of magnitude larger for noncoherent detection compared to differential detection. In general, the false alarm probability for differential detection is lower than that of the noncoherent 
scheme by the order of $1 / 2 \exp \left(V_{T_{n}} / 2\right)$ in very low ranges of SNR. Since the threshold is normalized to the thermal noise variance and the self noise is ignorable, all the curves tend to become flat in very low signal-to-noise ratios. In other words, the false alarm probability is independent of the signal level in this SNR range.

Under the hypothesis $H_{1}, Y_{k}$ is constant and is given by $Y_{k}=M \sqrt{E_{c} T_{c}}$. As a result, the decision variable is the sum of the product of two nonzero-mean and two zero-mean Gaussian random variables as follows:

$$
Z_{k}=\left(M \sqrt{E_{c} T_{c}}+N_{k}^{R}\right)\left(M \sqrt{E_{c} T_{c}}+N_{k-M}^{R}\right)+N_{k}^{I} N_{k-M}^{I} .
$$

Then, the detection probability can be written as

$$
P_{D}=\int_{V_{T}}^{\infty} f_{H_{1}}(\zeta) d \zeta=\int_{V_{T}}^{\infty} f_{2}(\zeta) * f_{3}(\zeta) d \zeta
$$

where $f_{2}(z)$ is as previously defined, and $f_{3}(z)$ is the density function of the nonzero-mean term. Equation (27) can be rewritten by normalizing the random variables by the thermal noise variance to obtain

$$
P_{D}=\int_{-\infty}^{\infty} f_{2 n}\left(V_{T_{n}}-\zeta\right) \int_{\zeta}^{\infty} f_{3 n}(\beta) d \beta d \zeta
$$

where $f_{2 n}$ and $f_{3 n}$ are the density functions of the normalized random variables. From (12), the expression for $f_{2 n}$ is

$$
f_{2 n}(z)=\frac{1}{\pi} K_{0}(|z|) .
$$

The inner integral, which corresponds to the detection probability of the nonzero-mean term, can be evaluated directly as follows:

$$
\Psi(\zeta)=\int_{\zeta}^{\infty} f_{3 n}(\beta) d \beta=\operatorname{Prob}(x y>\zeta)
$$

where $x$ and $y$ are the components of nonzero-mean Gaussian term normalized to the thermal noise variance. The above integral can be evaluated by calculating the volume surrounded by joint pdf of $x, y$ and the area $x y>\zeta$

$$
\begin{aligned}
\Psi(\zeta)= & \frac{1}{2 \pi} \\
& \times \int_{0}^{\infty} \int_{\frac{\zeta}{y}}^{\infty} \exp \left(-\frac{(x-m)^{2}+(y-m)^{2}}{2}\right) d x d y
\end{aligned}
$$

where $m=\sqrt{2 M \frac{E_{c}}{N_{0}}}$.

In the Appendix, the above is simplified to

$$
\begin{aligned}
\Psi(z)= & \frac{1+\operatorname{erf}\left(\frac{m}{\sqrt{2}}\right)}{4}-\frac{1}{\sqrt{8 \pi}} \int_{0}^{\infty} \exp \left(-\frac{(y-m)^{2}}{2}\right) \\
& \times \operatorname{erf}\left(\frac{-m+\frac{z}{y}}{\sqrt{2}}\right) d y .
\end{aligned}
$$

The final expression for the detection probability is given as

$$
P_{D}=\frac{1}{\pi} \int_{-\infty}^{\infty} K_{0}\left(V_{T_{n}}-\tau\right) \Psi(\tau) d \tau
$$

To find a fairly tight approximation for (33), we neglect the second term in (26). From (10), this approximation leads to the following density function for the decision variable under hypothesis $H_{1}$ :

$f_{H_{1}}(\zeta) \approx \frac{1}{2 \pi} \int_{0}^{\infty} \frac{1}{t} \exp \left(-\frac{1}{2}\left[\left(m-\frac{\zeta}{t}\right)^{2}+(m-t)^{2}\right]\right) d t$

After some manipulation, the detection probability is approximated as

$$
\begin{aligned}
P_{D} \approx & \Psi\left(V_{T_{n}}\right) \\
= & \frac{1+\operatorname{erf}\left(\frac{m}{\sqrt{2}}\right)}{4}-\frac{1}{\sqrt{8 \pi}} \int_{0}^{\infty} \exp \left(-\frac{(y-m)^{2}}{2}\right) \\
& \times \operatorname{erf}\left(\frac{-m+\frac{V_{T_{2 n}}}{y}}{\sqrt{2}}\right) d y .
\end{aligned}
$$

Clearly, the same result is obtained if we replace $\zeta$ in (30) by the normalized threshold $V_{T_{2}}$. Under hypothesis $H_{1}$ for noncoherent detection, the decision variable is the square of a Rician random variable, and the false alarm probability is then given as [3]

$$
P_{D}=\int_{V_{T_{\eta 2}}}^{\infty} \frac{1}{2} I_{0}(\sqrt{z} m) \exp \left(-\frac{z+m^{2}}{2}\right) d z .
$$

The probability of miss $P_{M}=1-P_{D}$ versus $\frac{E_{c}}{N_{0}}$ for $M=64$ and for two different normalized thresholds is plotted in Fig. 4. We observe that the difference in SNR between differential and noncoherent curves is more than 1 $\mathrm{dB}$ at high SNR, while this difference diminishes at lower SNR values. This observation is comparable with the DBPSK and noncoherent bit error probabilities in high signal-to-noise ratios. From the false alarm curves, we expect that for SNR values much lower than the practical range, i.e., for very low $M \frac{E_{c}}{N_{0}}$ values, the noncoherent detection slightly outperforms the differential scheme. This is because the independent multiplicative noise components play a more dominant role in detection probability, which results in poorer performance for differential detection in very low ranges of SNR.

It is also seen that the approximation of the detection probability given by (35) becomes tighter for larger threshold levels, as shown in Fig. 4. The reason for that stems from the behavior of $\Psi(\zeta)$, which flattens as the argument $\zeta$ increases. From (34), it is seen that the abruptly decreasing Hankel function $K_{0}$ symmetrically smoothes the $\Psi(\zeta)$ around the normalized threshold $V_{T_{n}}$. The function $\Psi(\zeta)$ flattens in higher $\zeta$ ranges, causing better approximation of the integral given in (35).

\section{DifFERENTIALly COHERENT PPC}

The structure of a differentially coherent DS acquisition receiver utilizing PPC is shown in Fig. 1(b), where MF1 and MF2 are matched to two consecutive subsequences, $c_{1}(t)$ and $c_{2}(t)$, of length $M$. The correct phase position of the received waveform is seen by MF1, $T$ seconds before it is seen by MF2. The matched filter outputs are given by

$$
Y_{k}^{i}=\sqrt{\frac{E_{c}}{T_{c}}} \int_{0}^{M T_{c}} c_{i}(t) c\left(t+\beta T_{c}+k T_{c}\right) d t, \quad i=1,2 .
$$




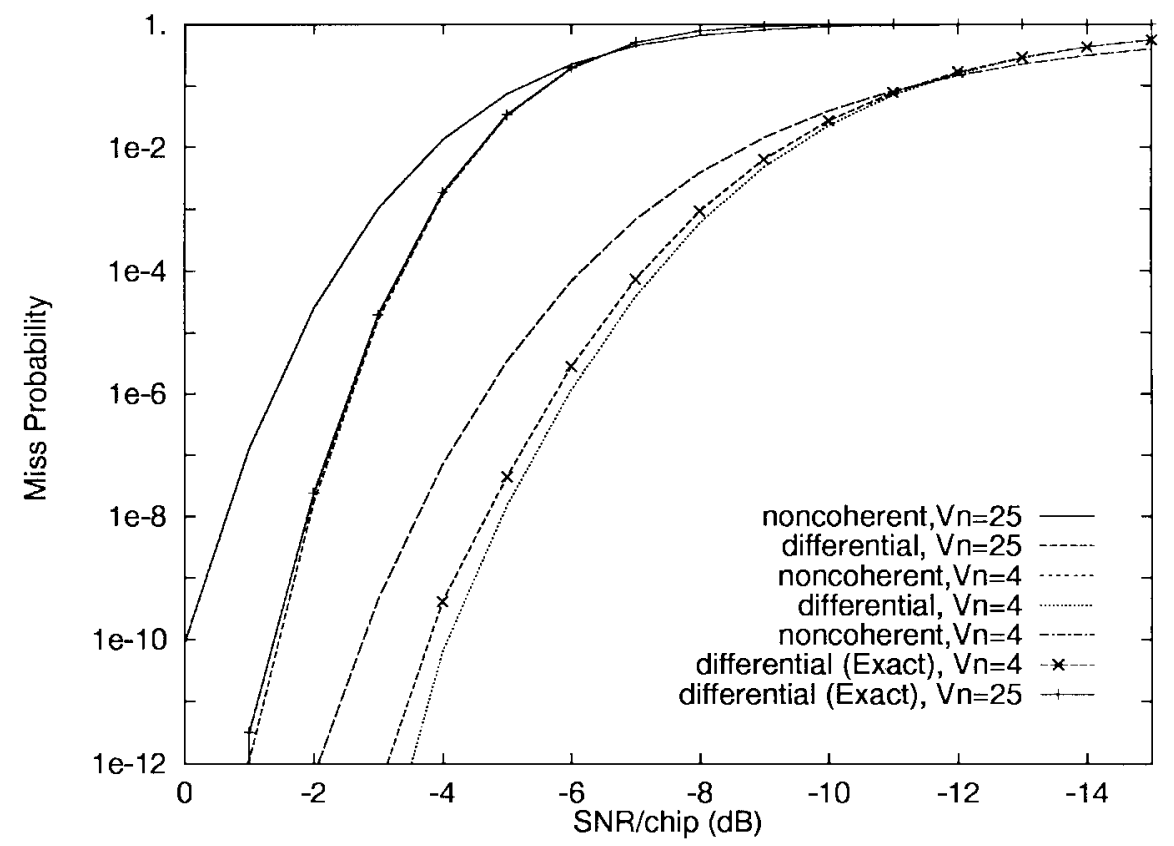

Fig. 4. Probability of miss versus chip SNR for PPC and FPC.

Under hypothesis $H_{1}$, the decision variable for this structure is the same as the one for the FPC receiver, because, as for FPC detection, the independent thermal noise components are the only random components which take part in the decision variable and no self-noise component is involved. Thus, $P_{D}$ would follow (35), which was derived for FPC.

Under hypothesis $H_{0}$, the decision variable is given as

$$
Z_{k}=\left(Y_{k}^{2}+N_{k}^{R, 2}\right)\left(Y_{k-M}^{1}+N_{k-M}^{R, 1}\right)+N_{k}^{I, 2} N_{k-M}^{I, 1}
$$

where $Y_{k-M}^{1}$ and $Y_{k}^{2}$ are the self-noise terms, and $\left(N_{k}^{R, i}, i=\right.$ $1,2)$ and $\left(N_{k-M}^{I, i}, i=1,2\right)$ are the real and imaginary parts of the thermal noise components of the two matched filters, respectively. The self-noise and thermal-noise components of the two matched filter outputs are clearly uncorrelated. Thus, for sufficiently large $M$, the self-noise components $Y_{k}^{2}$ and $Y_{k-M}^{1}$ are assumed independent Gaussian random variables. As a result, unlike with FPC detection, the two noise components in the first term of the decision variable are independent Gaussian random variables. The thermal noise parts at the MF outputs are also statistically independent and, consequently, the product of two independent zero-mean Gaussian r.v.s, with variance $\sigma_{Y}^{2}+\sigma_{n}^{2}=\sigma_{n}^{2}\left(1+\rho^{2}\right)$, forms the first term of the decision variable. Using (12), we obtain the pdf of the first term of the decision variable

$$
f_{4}(z)=\frac{1}{\pi \sigma_{n}^{2}\left(1+\rho^{2}\right)} K_{0}\left(\frac{|z|}{\sigma_{n}^{2}\left(1+\rho^{2}\right)}\right) .
$$

The density function for the second component of the decision variable is given by (12). Hence, the probability of false alarm can be written as

$$
P_{\mathrm{FA}}=\int_{V_{T}}^{\infty} f_{4}(\zeta) * f_{2}(\zeta) d \zeta
$$

After some manipulation, the false alarm probability becomes

$$
P_{\mathrm{FA}}=\frac{1}{\pi \sqrt{1+\rho^{2}}} \int_{-\infty}^{\infty} K_{0}\left(\frac{|\zeta|}{1+\rho^{2}}\right) \Phi\left(V_{T_{n}}-\zeta\right) d \zeta
$$

where $\Phi(\alpha)$ is defined by (16). The same approximation which was made in the FPC case can be applied to the probability of false alarm in the PPC case. In higher ranges of SNR values, the first term in (38) is dominant. Thus, the false alarm probability can be approximated as

$$
P_{\mathrm{FA}_{\mathrm{Asymp}},}^{H} \approx \int_{V_{T}}^{\infty} f_{4}(\zeta) d \zeta=\frac{1}{\pi} \int_{v^{*}}^{\infty} K_{0}(|\zeta|) d \zeta
$$

where

$$
v^{*}=\frac{V_{T_{n}}}{\left(1+\rho^{2}\right)} .
$$

Using (17), we can evaluate the integral in (42) as follows:

$$
\begin{aligned}
P_{\mathrm{FA}_{\text {Asymp. }}}^{H} \approx & \frac{1}{2}-\left[\frac{1}{\pi} v^{*} K_{0}\left(v^{*}\right)+\frac{1}{2} v^{*}\left\{L_{0}\left(v^{*}\right) K_{1}\left(v^{*}\right)\right.\right. \\
& \left.\left.+L_{1}\left(v^{*}\right) K_{0}\left(v^{*}\right)\right\}\right] .
\end{aligned}
$$

For lower SNR values, i.e., when $\rho$ tends to zero, we can ignore the self-noise component in the first term of (38). Hence, the thermal noise components play a dominant role in the decision variable. From (20), the false alarm probability is approximated as

$$
P_{\mathrm{FA}_{\text {Asymp }}}^{L} \approx \frac{1}{2} \int_{V_{T_{n}}}^{\infty} f_{H_{0}}(\zeta) d \zeta=\frac{1}{2} \exp \left(-V_{T_{n}}\right) .
$$

As expected, this equation is equivalent to (21). In very low SNR values, the thermal noise, which is a common term in both PPC and FPC, is the dominant factor in the false alarm probability and the self-noise components, which are different for the two detection schemes, are negligible. 


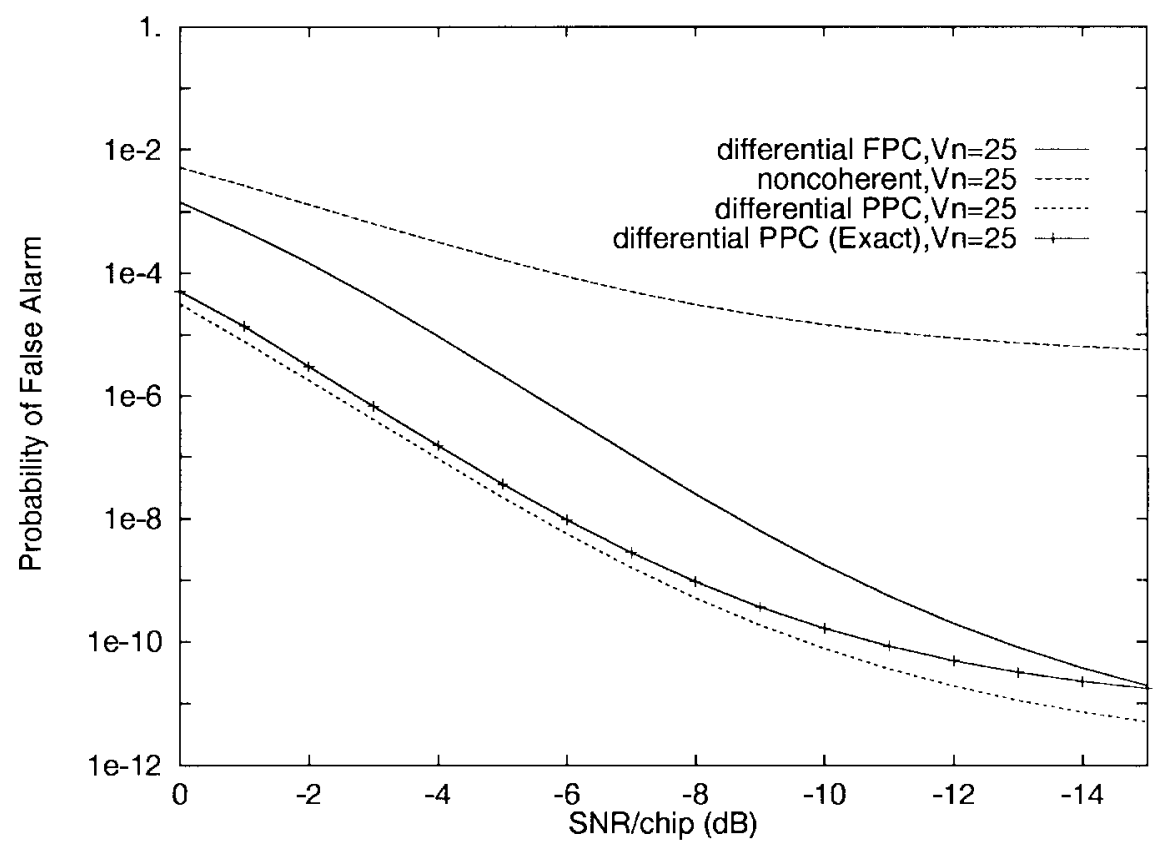

Fig. 5. Probability of false alarm versus chip SNR for PPC.

The false alarm probabilities for noncoherent and differentially-coherent PPC and FPC schemes are shown in Fig. 5. A major difference in $P_{\mathrm{FA}}$ among the three different schemes is observed. The lower false alarm probability in the PPC case is mainly due to independent thermal- and self-noise components in the decision variable. For lower values of SNR, the independent thermal-noise terms, which are common in both PPC and FPC cases, are dominant. Thus, the two differential curves would converge in this range of SNR, as analytically substantiated before. For higher SNR values, the self-noise components determine the false alarm probability. Thus, the noncoherent and differentially coherent FPC curves converge because of their correlated thermal noise components, whereas the differential PPC technique yields lower false alarm probability as a result of independent self-noise components.

\section{Single- And Multiple-Dwell AcQuisition Times}

We shall now evaluate the mean acquisition time for both PPC and FPC utilizing differential detection. Acquisition receivers are characterized as single-dwell or multiple-dwell, depending on whether the decision on acquisition is made on the basis of a single observation or many such observations. Single-dwell detectors can use either FPC or PPC. In contrast, multiple-dwell detectors almost invariably employ short-time PPC in the search mode, in order to expedite the acquisition process, since the negative effects of PPC can be removed in the verification mode [3].

In this section, the underlying system parameters, such as false alarm probability, detection probability, timing uncertainty interval, and the penalty time required to start a new search after a false alarm will be translated into the mean acquisition time, which is a more meaningful acquisition parameter. We will compare the mean acquisition time for differential FPC, differential PPC, and noncoherent detection in both single- and multiple-dwell schemes. Both PPC and FPC are considered in the single-dwell scheme. For multiple-dwell detection, only the PPC case is investigated.

For a single-dwell receiver, one can arrive at the following expression using the generating function flow graph method [1]:

$$
\frac{E\left(T_{\mathrm{acq}}\right)}{\tau_{d}}=\frac{2+\left(2-P_{d}\right)(q-1)\left(1+J P_{\mathrm{fa}}\right)}{2 P_{d}}
$$

where $q$ is the total number of decision samples in the uncertainty region, $\tau_{d}$ is the dwell time, and $J \gg 1$ is the number of penalty time units due to false alarm.

With multiple-dwell detection, the search mode is followed by a verification algorithm, in order to avoid a costly false alarm that can supply the tracking system with a wrong phase. The verification mode utilized here uses a majority logic type of decision on the total set of multiple-dwell threshold tests. If $B$ out of $A$ samples exceed a threshold $\gamma$, acquisition is declared, and the tracking system is enabled. For a multipledwell receiver with nonabsorbing false alarm state, the mean acquisition time is given by

$$
\frac{E\left(T_{\mathrm{acq}}\right)}{\tau_{d}}=\frac{1+K P_{d 1}+(q-1) T_{\mathrm{fa}}\left(1-P_{d 1} P_{d 2} / 2\right)}{P_{d 1} P_{d 2}}
$$

where

$$
T_{\mathrm{fa}}=1+K P_{\mathrm{fa} 1}+J P_{\mathrm{fa} 1} P_{\mathrm{fa} 2}
$$

$J$ is the false alarm penalty time, $A$ and $B$ are the verification mode parameters, and $\tau_{d}$ and $K \tau_{d}$ are the delays in the first and second dwell times, respectively. For a dwell time of one chip period, which is assumed here, $K=A M$. 


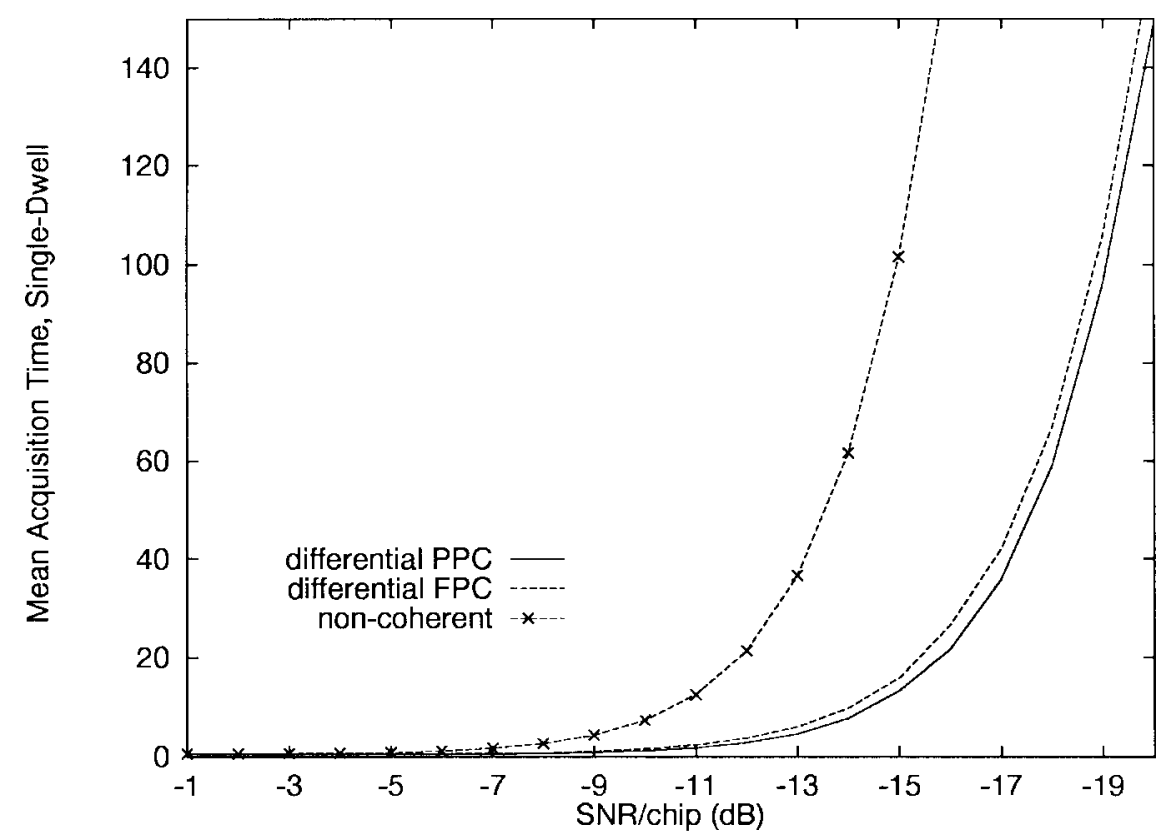

Fig. 6. Mean acquisition time versus chip SNR for single-dwell detector.

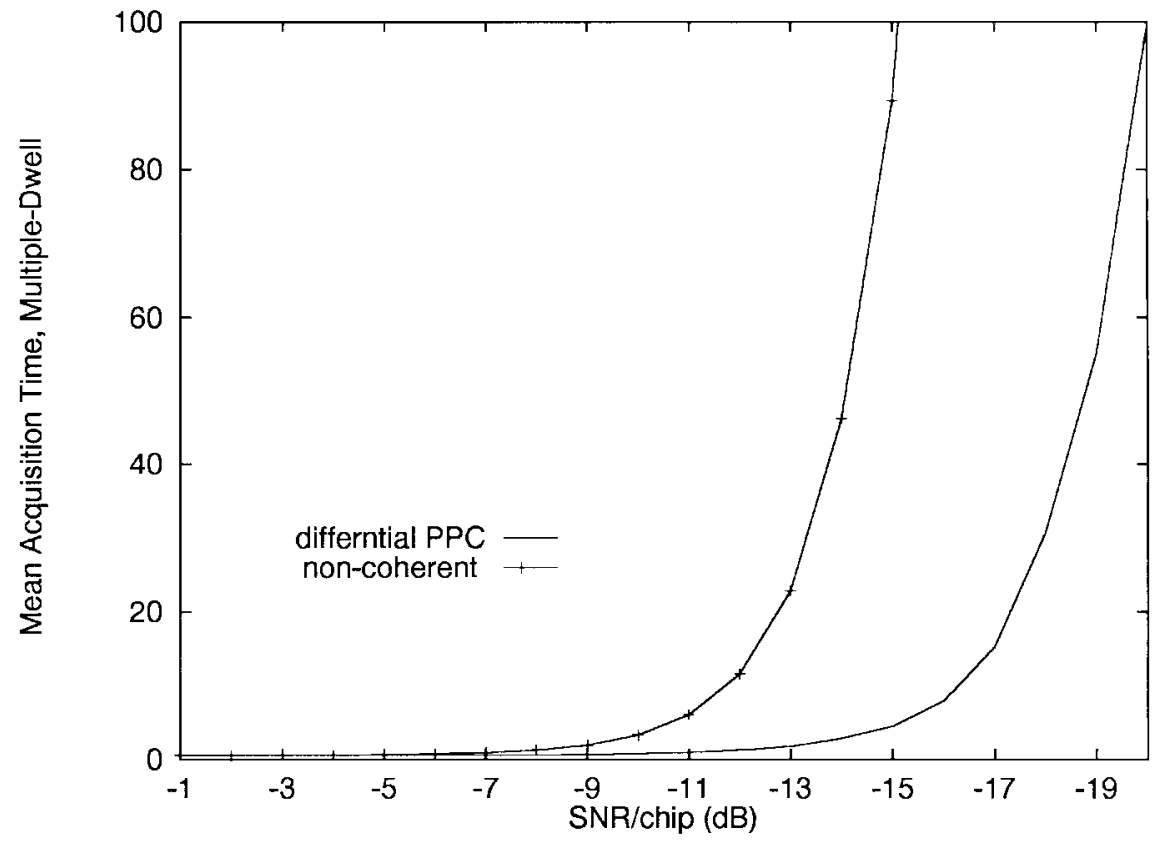

Fig. 7. Mean acquisition time versus chip SNR for multiple-dwell detector.

\section{RESULTS AND DISCUSSION}

Differentially coherent detection was applied to a system with full code period uncertainty region and the advancing step of one chip period. The number of integration chips is considered to be $M=64$, which is also assumed as the uncertainty period for the FPC scheme. The uncertainty region of $r=2^{15}-1$ chips (IS-95 proposal [16]) is considered for the PPC algorithm. We assume that the penalty time is 100 times greater than the number of integration chips in FPC and greater than the verification time in PPC by the same factor.

Fig. 6 shows the the mean acquisition time versus SNR per chip for a single-dwell detector with $M=64$ and
$J=100 M$. The optimal threshold which yields the minimum mean acquisition time has been calculated for each SNR value, and the corresponding acquisition time is shown in Fig. 7. The mean acquisition time is normalized to the uncertainty period in both PPC and FPC cases. The three curves correspond to noncoherent, differentially coherent PPC, and differentially coherent FPC detection schemes. It is observed that a minimal permissible input SNR exists, below which the system rapidly deteriorates. This minimal value, however, greatly varies from one scheme to the other. Over the whole range of SNR values, the differential scheme yields more than 4-5 dB improvement in SNR over the noncoherent scheme. It is also shown that 
the PPC scheme slightly outperforms the FPC scheme, due to its better detection performance. The two curves converge in lower values of SNR, as shown in the figure. This is expected, since in this range of SNR, thermal noise is the dominant factor in the acquisition parameters. It is seen that the remarkable difference between the performances of the differential and noncoherent schemes is preserved almost in the whole range of desirable SNR values. It was also observed that, by changing the false alarm penalty time $J \tau_{d}$, the same $4-\mathrm{dB}$ difference between the noncoherent and differential curves is maintained.

For multiple-dwell detection, $A=4$ and $B=2$ are suggested in [3] as reasonable choices for verification parameters. Other parameters include the number of integration chips $M=64$, the number of time units in confirmation mode $K=A M$, the false alarm penalty time $J=100 K$, and the uncertainty region of $r=2^{15}-1$ chips.

The normalized mean acquisition times versus $E_{c} / N_{0}$ for differential PPC and noncoherent detection are plotted in Fig. 7. Since FPC detection is not commonly used in multipledwell schemes, only a PPC receiver was investigated. From Fig. 7, we observe that the differential PPC scheme outperforms the conventional noncoherent scheme by a margin of $5 \mathrm{~dB}$ in SNR. This striking improvement is mainly a result of the large difference in false alarm probabilities of the two schemes at low SNR values.

It should be noted that optimum threshold setting must be incorporated in the evaluation of the mean acquisition for each SNR value. In other words, for each SNR value, there is an optimal threshold which minimizes the multiple-dwell mean acquisition time. In order to plot the curves in Figs. 6 and 7, the optimal threshold has been employed in both noncoherent and differential detection schemes.

\section{CONCLUSION}

In this paper, we have proposed a differential detection scheme for the acquisition of DS/BPSK signals and evaluated its performance. Closed-form expressions for the detection and false alarm probabilities were obtained for the cases of FPC and PPC, and the corresponding mean acquisition times for single- and multiple-dwell detectors were evaluated. The differential detection scheme gives a remarkable improvement in performance over the conventional noncoherent scheme. An improvement by a margin of 4-5 dB in SNR for both singleand multiple-dwell detectors is attained.

\section{APPENDIX}

To calculate the pdf of the product of two correlated r.v., we substitute (9) in (10)

$$
\begin{aligned}
f_{1}(z)= & \frac{1}{2 \pi \sigma_{n} \sqrt{2 \sigma_{Y}^{2}+\sigma_{n}^{2}}} \\
& \cdot \int_{-\infty}^{\infty} \frac{1}{w} \exp \left(-\frac{\left(w-\frac{z}{w}\right)^{2}}{4 \sigma_{n}^{2}}-\frac{\left(w+\frac{z}{w}\right)^{2}}{8 \sigma_{Y}^{2}+4 \sigma_{n}^{2}}\right) d w .
\end{aligned}
$$

Further manipulations and replacing $\frac{\sigma_{Y}}{\sigma_{\eta 2}}$ with $\rho$ yields

$$
\begin{aligned}
f_{1}(z)= & \frac{1}{\pi \sigma_{n}^{2} \sqrt{1+2 \rho^{2}}} \exp \left(\frac{\rho^{2} z}{\sigma_{n}^{2}\left(1+2 \rho^{2}\right)}\right) \\
& \cdot \int_{0}^{\infty} \frac{1}{w} \exp \left(-\left(w^{2}+\frac{z^{2}}{w^{2}}\right)\left(\frac{1}{2 \sigma_{n}^{2}} \frac{1+\rho^{2}}{1+2 \rho^{2}}\right)\right) d w .
\end{aligned}
$$

Equation (50) can be rephrased to

$$
\begin{aligned}
f_{1}(z)= & \frac{1}{2 \pi \sigma_{n}^{2} \sqrt{1+2 \rho^{2}}} \exp \left(\frac{\rho^{2} z}{\sigma_{n}^{2}\left(1+2 \rho^{2}\right)}\right) \\
& \cdot \int_{0}^{\infty} \frac{1}{\zeta} \exp \left(-\frac{\alpha}{2}\left(\zeta+\frac{z^{2}}{\zeta}\right)\right) d \zeta
\end{aligned}
$$

where $\alpha=\frac{1}{2 \sigma_{n}^{2}} \frac{1+\rho^{2}}{1+2 \rho^{2}}$. The integral in the right side of (51) is an integral representation of $K_{0}(\alpha z)$, as given in [14]. Thus, (11) is directly obtainable from the above expression.

In the absence of self noise, the decision variable consists of two multiplicative thermal noise with the same pdf, i.e., $p(z)=\frac{1}{\pi \sigma_{n}^{2}} K_{0}\left(\frac{|z|}{\sigma_{\eta}^{2}}\right)$.

To substantiate the expression in (20), we use the Fourier transform of the convolution. From [15], the Fourier transform of $p(z)$ is given by

$$
\mathcal{F}\left(K_{0}(a z)\right)=\frac{\pi}{\sqrt{a^{2}+\omega^{2}}}
$$

Thus, the transform of the decision variable which is the convolution of two Hankel functions can be given by

$$
\begin{aligned}
\mathcal{F}\left(f_{H_{0}}(z)\right) & =\mathcal{F}\left(\frac{1}{\pi \sigma_{n}^{2}} K_{0}\left(\frac{|z|}{\sigma_{n}^{2}}\right) * \frac{1}{\pi \sigma_{n}^{2}} K_{0}\left(\frac{|z|}{\sigma_{n}^{2}}\right)\right) \\
& =\frac{1}{\sigma_{n}^{4}} \frac{1}{\left(\frac{1}{\sigma_{n}^{4}}+\omega^{2}\right)} .
\end{aligned}
$$

Taking the inverse Fourier transform from the right side of (53), the spectral density of the decision variable is obtained

$$
f_{H_{0}}(z)=\frac{1}{2 \sigma_{n}^{2}} \exp \left(-\frac{|z|}{\sigma_{n}^{2}}\right) .
$$

Under hypothesis $H_{1}$, the detection probability given in (35) can be evaluated by directly manipulating the double integral in (31)

$$
\begin{aligned}
\Psi(z)= & \frac{1}{2 \pi} \int_{0}^{\infty} \exp \left(-\frac{(y-m)^{2}}{2}\right) \\
& \cdot\left(\int_{\frac{z}{y}}^{\infty} \exp \left(-\frac{(x-m)^{2}}{2}\right) d x\right) d y \\
= & \frac{1}{2 \pi} \int_{0}^{\infty} \sqrt{\frac{\pi}{2}}\left(1-\operatorname{erf}\left(\frac{-m+\frac{z}{y}}{\sqrt{2}}\right)\right) \\
& \cdot \exp \left(-\frac{(y-m)^{2}}{2}\right) d y
\end{aligned}
$$

which finally leads to (35). 


\section{REFERENCES}

[1] M. K. Simon, J. K. Omura, R. A. Scholtz, and B. Levitt, SpreadSpectrum Communications. Rockville, MD: Computer Science, 1985.

[2] J. K. Holmes and C. C. Chen, "Acquisition time performance of PN spread-spectrum systems," IEEE Trans. Commun., vol. COM-25, pp. 778-783, Aug. 1977.

[3] A. Polydoros and C. L. Weber, "A unified approach to serial-search spread-spectrum code acquisition-Part II: A matched-filter receiver," IEEE Trans. Commun., vol. COM-32, pp. 550-560, May 1984.

[4] S. S. Rappaport and D. M. Grieco, "Spread-spectrum acquisition: Methods and technology," IEEE Commun. Mag., vol. 22, no. 6, pp. 6-21, June 1984

[5] L. B. Milstein, J. Gevargiz, and P. K. Das, "Rapid acquisition for direct sequence spread spectrum communications using parallel SAW convolvers," IEEE Trans. Commun., vol. COM-33, pp. 593-600, July 1985.

[6] S. G. Glisic, "Automatic decision threshold level control in direct sequence spread spectrum systems based on matched filtering," IEEE Trans. Commun., vol. 36, pp. 519-527, Apr. 1988.

[7] K. M. Cheung, H. Leib, and S. Pasupathy, "Multipath induced intersymbol interference bandwidth expanding signaling," in Proc. 16th Biennial Symp. Communications, May 1992, pp. 273-276.

[8] M. H. Zarrabizadeh and E. S. Sousa, "Analysis of a differentially coherent spread-spectrum acquisition receiver," in Proc. 1995 Int. Conf. Communications (ICC'95), pp. 1732-1736.

[9] _ "Analysis of a differentially coherent DS-SS parallel acquisition receiver," in Proc. 1995 Vehicular Technol. Conf. (VTC'95), pp. 271-275.

[10] E. A. Geraniotis, "Noncoherent hybrid DS-SFH spread-spectrum multiple-access communications," IEEE Trans. Commun., vol. COM-34, pp. 862-872, Sept. 1986

[11] A. J. Viterbi, "The evolution of digital wireless technology from space to personal communication services," IEEE Trans. Commun., vol. COM26, pp. 1689-1702, Nov. 1978.

[12] W. H. Huggins, "Signal-flow graphs and random signals," in Proc. IRE, vol. 45, pp. 74-86, Jan. 1957.

[13] A. Papoulis, Probability, Random Variables, and Stochastic Processes, 3rd ed. New York: McGraw-Hill, 1991.

[14] I. S. Gradshteyn and I. M. Ryzhik, Table of Integrals, Series, and Products. New York: Academic, 1980.

[15] M. Abramowitz and I. A. Stegun, Handbook of Mathematical Functions With Formulas, Graphs, and Mathematical Tables, National Bureau of Standards Applied Mathematics Series 55, Washington, DC, 1964.

[16] Mobile Station-Base Station Compatibility Standard for Dual-Mode Wideband Spread Spectrum Cellular System, TIA/EIA Standard IS-95, 1995.

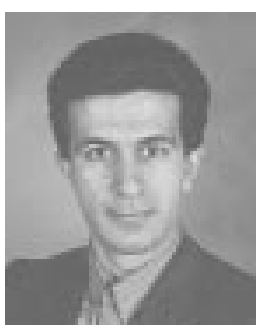

Mohammad H. Zarrabizadeh was born in Yazd, Iran, in 1961. He received the B.Sc. and M.Sc. degrees from the University of Tehran, Tehran, Iran, in 1986 and 1989, respectively, and the Ph.D. degree from the University of Toronto, Toronto, Ont., Cananda, in 1996, all in electrical engineering.

He is currently a Member of the Technical Staff at Lucent Technologies Bell Laboratories, Holmdel, NJ, working on digital broadcating and cellular technologies. His research interests include digital communcations, high-throughput data transmission, and synchronization of wireless communcation systems.

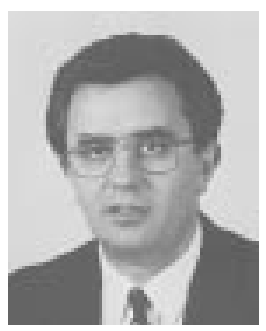

Elvino S. Sousa (S'79-M'80-SM'96) was born in Graciosa, The Azores, Portugal, in 1956. He received the B.A.Sc. degree in engineering science and the M.A.Sc. degree in electrical engineering from the University of Toronto, Toronto, Ont., Canada, in 1980 and 1982, respectively, and the Ph.D. degree in electrical engineering from the University of Southern California, Los Angeles, in 1985.

Since 1986, he has been with the Department of Electrical and Computer Engineering, University of Toronto. From 1986 to 1996, he held the ranks of Assistant and Associate Professor and was also a Natural Sciences and Engineering Research Council (NSERC) University Research Fellow. In 1997, he was promoted to Professor. Since 1996, he has also been the Chair of the Communications Group. He has performed research in spread-spectrum systems since 1983. His current interests are in the areas of spread-spectrum systems, mobile communications, indoor wireless communications, and wireless LAN's. At the University of Toronto, he teaches graduate courses in mobile communications and error control codes and is the Leader of the Wireless Communications Laboratory. He has been invited to give lectures and short courses on spread-spectrum and wireless communications in a number of different countries.

Dr. Sousa is an Associate Editor in the area of CDMA systems for the IEEE Transactions on Communications. He was also the Technical Program Chairman for the 6th IEEE International Symposium on Personal, Indoor and Mobile Radio Communications (PIMRC 95), held in Toronto, Canada, in September 1995 . 